\title{
Quantum dissipative systems from theory of continuous measurements
}

\author{
Michael B. Mensky \\ P.N.Lebedev Physics Institute, 117924 Moscow, Russia \\ Stig Stenholm \\ Royal Institute of Technology (KTH), Stockholm
}

\begin{abstract}
We apply the restricted-path-integral (RPI) theory of non-minimally disturbing continuous measurements for correct description of frictional Brownian motion. The resulting master equation is automatically of the Lindblad form, so that the difficulties typical of other approaches do not exist. In the special case of harmonic oscillator the known familiar master equation describing its frictionally driven Brownian motion is obtained. A thermal reservoir as a measuring environment is considered.
\end{abstract}

\section{Introduction}

Irreversible behavior is an every day phenomenon in physics, even if the basic theories have simple properties under time reversal. It has long been realized, that in certain limits, an incoherent environment will provide a reservoir for relaxation processes, which in thermal equilibrium will enforce the proper distribution functions. More recently, it has been realized that also observations by measurements introduce decoherence, which from the point of view of the system evolution is equivalent to environmental dephasing. A comprehensive summary of the recent progress in this field is found in Ref. [1]. 
In the case of a continuous but weak observation, we can design quantum probability amplitudes conditioned on the continuously observed variable. Such a system can be formulated in a natural way by putting restrictions on the path integral providing the quantum propagator of the system. Such restricted path integrals (RPI) have been found to clarify many questions related to the continuous monitoring of a selected variable [2]. In particular, it can incorporate the unavoidable disturbing effect of the observation back on the system observed. This is termed a minimally disturbing measurement. Non-minimally disturbing continuous measurements have also been suggested [2]. In this paper we pursue the consequences of a simple model for such a measurement.

The RPI approach is basically selective. This means that the measurement readout, or history of observations, is taken into account so that the dynamics of the measured system conditioned by this history is presented by a state vector. It is possible to connect the path integral formulation to the more common master equation approach [2]. This is done by going over to the non-selective description of the continuous measurement. If we take the time evolution conditioned by the given measurement readout (history of observations), express it in terms of a density matrix and then perform a functional summation over all possible observed histories, we obtain an ensemble description comprising all possible histories of observations. The resulting total density matrix obeys a master equation.

The approach to environmental influences making use of master equations goes back a long time, but the recent interest has been kindled by the works of Caldeira and Leggett, Zurek, Unruh and Zurek [3]. In many connections, especially those related to quantum optics, these results are satisfactory, but when applied to frictional Brownian motion they are found to be flawed [四; see also the discussions in [5]. The reason is that they are not of the Lindblad form, and hence they cannot preserve the positivity of the density matrix. It is important that this is the case even if the fluctuation-dissipation theorem is fulfilled. Some authors claim that this is of little consequence, but it does signal a possible danger in applications. The point is that even correctly derived master equations fulfilling the fluctuation-dissipation theorem, may not be of the Lindblad form [6]. A discussion of the situation is found in Ref. "77.

Here we apply the RPI theory of continuous measurements to obtain correct description of frictional Brownian motion. We formulate the RPI de- 
scription for a non-minimally disturbing continuous observation. Obtaining the phenomenological description of the measurement (including its backaction on the measured system), we go over to the non-selective description and investigate the resulting master equation. It turns out to be of Lindblad form for any choice of the parameters of the measurement.

Then we derive the equations of motion for the first and second moments which turn out to nearly coincide with the expected Ornstein-Uhlenbeck equations. The main correction is a position diffusion coefficient which may be derived from the quantum uncertainty relation. The conditions are found when this term may be omitted. In case of thermal equilibrium of the measured system with thermal reservoir, the strength of the continuous measurement performed by the reservoir is shown to depend on the reservoir's temperature. If, however, the interaction between the system and reservoir is weak enough, the equilibrium turns out to be impossible, and the relation between the strength of the measurement and the temperature is violated.

The paper is organized in the following way. In Sect. 2 we formulate the RPI description of a (non-minimally disturbing) continuous measurement, derive the corresponding master equation and show that it automatically turns out to be of the Lindblad type. In Sect. 3 we restrict our treatment to the familiar case of a harmonic oscillator. We find that continuously monitoring the momentum of the oscillator (by its environment) leads to the familiar form of the master equation [7] describing the frictionally driven Brownian motion. This gives the Ornstein-Uhlenbeck-type equations for the first and second moments. In Sect. 4the equilibrium of the measured system with the (measuring) reservoir having a definite temperature is discussed. Finally Sect. 5 summarizes and concludes our paper.

\section{Non-minimally disturbing measurement}

The restricted path integral (RPI) for the monitoring of a quantum observable $A(q, p)$ with the observed history $a(t)$ may be formally written [2] as an ordinary Feynman path integral (in the phase-space representation) with the weight functional of the form

$$
w_{0}[a]=\exp \left\{\int d t\left[-\kappa(A(q, p)-a(t))^{2}\right]\right\}
$$


included in the itegrand. In case of non-minimally disturbing monitoring, imaginary terms are also present in the exponent [2]. In the linear approximation this gives

$$
w[a]=\exp \left\{\int d t\left[-\kappa(A(q, p)-a(t))^{2}-\frac{i}{\hbar}(\lambda a(t) B(q, p)+C(q, p))\right]\right\}
$$

Here $\kappa$ and $\lambda$ are the real parameters which characterize correspondingly the strength of the measurement (monitoring) and non-minimal disturbance produced by it. The real term in the exponent leads to the restriction of the path integral that is analogous to von Neumann's projecting in case of instantaneous measurements and is necessary for observations. Imaginary terms are responsible for adding phase in the course of the measurement which is not necessary for the observation, hence the terminology 'non-minimally disturbing measurement'.

With these definitions, the time evolution conditioned on the observation of $a(t)$ can be written as a RPI

$$
\begin{aligned}
U_{[a]}\left(q, q^{\prime}, t\right)= & \int d[p] \int_{q}^{q^{\prime}} d[q] \exp \left\{\int _ { 0 } ^ { t } d t \left[\frac{i}{\hbar}(p \dot{q}-H(q, p))\right.\right. \\
& \left.\left.-\kappa(A(q, p)-a(t))^{2}-\frac{i}{\hbar}(\lambda a(t) B(q, p)+C(q, p))\right]\right\} .
\end{aligned}
$$

The corresponding evolution operator $U_{[a]}(t)$ allows one to express the density matrix at an arbitrary time conditioned on the observation of $a(t)$ :

$$
\hat{\rho}_{[a]}(t)=U_{[a]}(t) \hat{\rho}(0) U_{[a]}^{\dagger}(t) .
$$

The ensemble averaged or total density matrix is obtained from this by carrying out the functional integration over all possible observations (measurement readouts) $[a]$ :

$$
\hat{\rho}(t)=\int d[a] \hat{\rho}_{[a]}(t)
$$

As a result we obtain

$$
\begin{aligned}
\hat{\rho}(t) & =\int d\left[q^{\prime}, p^{\prime}\right] \int d\left[q^{\prime \prime}, p^{\prime \prime}\right] \rho_{0}\left(q^{\prime}, q^{\prime \prime}\right) \\
& \times \exp \left[\int _ { 0 } ^ { t } d t \left(\frac{i}{\hbar}\left(p^{\prime} \dot{q}^{\prime}-H^{\prime}-C^{\prime}\right)-\frac{i}{\hbar}\left(p^{\prime \prime} \dot{q}^{\prime \prime}-H^{\prime \prime}-C^{\prime \prime}\right)\right.\right. \\
& \left.-\frac{\kappa}{2}\left(A^{\prime}-A^{\prime \prime}\right)^{2}-\frac{\lambda^{2}}{8 \kappa \hbar^{2}}\left(B^{\prime}-B^{\prime \prime}\right)^{2}+\frac{i \lambda}{2 \hbar}\left(A^{\prime}+A^{\prime \prime}\right)\left(B^{\prime \prime}-B^{\prime}\right)\right]
\end{aligned}
$$


where the notations $A^{\prime}=A\left(q^{\prime}, p^{\prime}\right), B^{\prime \prime}=B\left(q^{\prime \prime}, p^{\prime \prime}\right)$ etc. are used.

From this we can calculate the master equation for the density matrix by taking the time derivative and noting that all primed operators go to the left of $\hat{\rho}$ and the doubly primed ones to the right.

We still need to resolve the order of $\hat{A}$ and $\hat{B}$ when taken at the same time. Our physical interpretation suggests a solution: as $\hat{B}$ is the back action of observing $\hat{A}$, the latter should act before the former. We thus obtain the equation

$$
\begin{aligned}
\frac{\partial}{\partial t} \hat{\rho}= & -\frac{i}{\hbar}[\hat{H}+\hat{C}, \hat{\rho}]-\frac{\kappa}{2}[\hat{A},[\hat{A}, \hat{\rho}]]-\frac{\lambda^{2}}{8 \kappa \hbar^{2}}[\hat{B},[\hat{B}, \hat{\rho}]] \\
& -\frac{i \lambda}{2 \hbar}\left[\hat{B},[\hat{A}, \hat{\rho}]_{+}\right] .
\end{aligned}
$$

Let us rewrite the master equation (77) by introducing the operator

$$
\hat{l}=\hat{A}-i \frac{\lambda}{2 \kappa \hbar} \hat{B}
$$

Solving for $\hat{A}$ and $\hat{B}$ and inserting into Eq. (7) we obtain

$$
\begin{aligned}
\frac{\partial}{\partial t} \hat{\rho}= & -\frac{i}{\hbar}\left[\hat{H}+\hat{C}-i \frac{\kappa \hbar}{4}\left(\hat{l}^{\dagger 2}-\hat{l}^{2}\right), \hat{\rho}\right] \\
& -\frac{\kappa}{2}\left(\hat{l}^{\dagger} \hat{l} \hat{\rho}-2 \hat{l} \hat{\rho} \hat{l}^{\dagger}+\hat{\rho} \hat{l} \hat{l}^{\dagger}\right)
\end{aligned}
$$

The resulting equation is of the Lindblad form. The original Hamiltonian of the measured system is renormalized by the measurement procedure.

\section{Special case of a harmonic oscillator}

We shall now specialize to the case of a harmonic oscillator,

$$
\hat{H}=\frac{\hat{P}^{2}}{2}+\frac{1}{2} \omega^{2} \hat{Q}^{2} .
$$

Let the momentum operator be monitored and the coordinate operator presents non-minimal disturbance (this means that the momentum is shifted when being monitored):

$$
\hat{A}=\hat{P}, \quad \hat{B}=\omega \hat{Q}
$$


The weight factor (2) takes the form

$$
w[a]=\exp \left\{\int d t\left[-\kappa(p-a(t))^{2}-\frac{i}{\hbar}(\lambda \omega a(t) q+C(q, p))\right]\right\} .
$$

Exponential of operator $\hat{Q}$ is the displacement operator for observable $\hat{P}$. Therefore, the non-minimal disturbance (determined by the term proportional to $q$ in Eq. (12)) consists in this model in shifting the momentum (the same observable which is measured).

The master equation now becomes (for $\hat{C}=0$ )

$$
\begin{aligned}
\frac{\partial}{\partial t} \hat{\rho}= & -\frac{i}{\hbar}[\hat{H}, \hat{\rho}]-\frac{\kappa}{2}[\hat{P},[\hat{P}, \hat{\rho}]]-\frac{\lambda^{2} \omega^{2}}{8 \kappa \hbar^{2}}[\hat{Q},[\hat{Q}, \hat{\rho}]] \\
& -\frac{i \lambda \omega}{2 \hbar}\left[\hat{Q},[\hat{P}, \hat{\rho}]_{+}\right] .
\end{aligned}
$$

This is the well known master equation for a Brownian motion [7]. The Brownian motion of the oscillator is thus interpreted as the effect of monitoring the momentum by a continuously acting environment (reservoir).

The equations for the first moments (mean values of $\hat{P}$ and $\hat{Q}$ ) following from Eq. (13) are

$$
\begin{aligned}
\left\langle\frac{\partial \hat{P}}{\partial t}\right\rangle & =-\omega^{2}\langle\hat{Q}\rangle-\lambda \omega\langle\hat{P}\rangle \\
\left\langle\frac{\partial \hat{Q}}{\partial t}\right\rangle & =\langle\hat{P}\rangle
\end{aligned}
$$

and the equations for the second moments

$$
\begin{aligned}
\frac{\partial}{\partial t}\left\langle\hat{P}^{2}\right\rangle & =-\omega^{2}\langle\hat{P} \hat{Q}+\hat{Q} \hat{P}\rangle-2 \lambda \omega\left\langle\hat{P}^{2}\right\rangle+\frac{\lambda^{2} \omega^{2}}{4 \kappa} \\
\frac{\partial}{\partial t}\langle\hat{P} \hat{Q}+\hat{Q} \hat{P}\rangle & =-\lambda \omega\langle\hat{P} \hat{Q}+\hat{Q} \hat{P}\rangle+2\left(\left\langle\hat{P}^{2}\right\rangle-\omega^{2}\left\langle\hat{Q}^{2}\right\rangle\right) \\
\frac{\partial}{\partial t}\left\langle\hat{Q}^{2}\right\rangle & =\langle\hat{P} \hat{Q}+\hat{Q} \hat{P}\rangle+\kappa \hbar^{2} .
\end{aligned}
$$

We can introduce a damping constant in Eqs.(14) and (15) by writing $\gamma=\lambda \omega$. Equations (15) are appropriate for an Ornstein-Uhlenbeck process except for the diffusion term in position space. The same type of equations follow from 
the model introduced by Gallis [8]; see also [7]. Also Diósi [9] has pointed out that such a term is necessary to obtain a Lindblad form of evolution.

In the classical limit, $\hbar \rightarrow 0$, Eq. (15) gives the steady-state solution

$$
\langle\hat{P} \hat{Q}+\hat{Q} \hat{P}\rangle=0, \quad\left\langle\hat{P}^{2}\right\rangle=\omega^{2}\left\langle\hat{Q}^{2}\right\rangle=\frac{\lambda \omega}{8 \kappa}
$$

which agrees with the virial theorem. These result in the expression for the mean energy

$$
\langle\hat{H}\rangle=\frac{1}{2}\left\langle\hat{P}^{2}+\omega^{2} \hat{Q}^{2}\right\rangle=\frac{\lambda \omega}{8 \kappa} .
$$

The additional diffusion term in the third of Eqs. (15) has got no simple physical interpretation within a classical stochastic model. However, it is easily interpreted in the scheme of measurements as a consequence of the uncertainty relations. The fact that the term is proportional to $\hbar^{2}$ signals its quantum origin. Indeed, with time passing, momentum is measured with better precision, and therefore the uncertainty of the coordinate becomes larger. This may be shown to be expressed by just this term; see [10] and [2, Sect. 4.3.4] for the dual situation when the coordinate is continuously measured and grows more uncertain.

This diffusion term causes a spreading of the position variable according to $\Delta\left\langle\hat{Q}^{2}\right\rangle \approx \kappa \hbar^{2} t$. If this is required to be much smaller than the steady state value in (16), we find that its effect is negligible if

$$
t \ll \frac{\lambda}{8 \kappa^{2} \omega \hbar^{2}}
$$

\section{Thermal reservoir as an environment}

Let us assume that the environment of our (measured) oscillator is a thermal bath at some temperature $T$ and the oscillator is in equilibrium with the environment. Then the density matrix of the oscillator corresponds to the Boltzmann distribution in energies, which may be used for calculating mean

value of the number of energy level $\hat{n}=a^{\dagger} a$ and therefore mean value of energy:

$$
\bar{n}=\frac{1}{\exp \left(\hbar \omega / k_{B} T\right)-1}, \quad\langle\hat{H}\rangle=\hbar \omega\left\langle\hat{n}+\frac{1}{2}\right\rangle=\frac{\hbar \omega}{2} \operatorname{coth} \frac{\hbar \omega}{2 k_{B} T}
$$


This provides the fluctuation-dissipation theorem which relates the momentum diffusion coefficient and friction coefficient in the form

$$
D=\frac{\gamma \hbar \omega}{2} \operatorname{coth}\left(\frac{\hbar \omega}{2 k_{B} T}\right) \text {. }
$$

This gives in our case

$$
\lambda=4 \kappa \hbar \operatorname{coth}\left(\frac{\hbar \omega}{2 k_{B} T}\right) .
$$

Therefore, the strength of non-minimal disturbance depends on temperature of the measuring reservoir.

From Eq. (21) the inequality

$$
\lambda>4 \hbar \kappa
$$

follows. We see from this inequality that for $\lambda>4 \hbar \kappa$, the oscillator may be in thermal equilibrium with the reservoir. It can be shown that the regime of measurement is in this case classical, quantum effects are negligible.

The question naturally arises as to what happens if the non-minimal disturbance of the measurement is small enough (in comparison with the strength of the measurement) so that $\lambda<4 \hbar \kappa$. In this case the measurement is performed in quantum regime, but the situation seems to contradict the inequality (22). However, we have derived this inequality under the assumption that the distribution of the oscillator over energies is of the Boltzmann form, i.e. the oscillator is in equilibrium with the thermal reservoir. If this inequality is violated, thermal equilibrium of the oscillator with the thermal reservoir is impossible.

All these conclusions are made in the framework of the certain model of the continuous measurement. In this model the momentum is continuously monitored and non-minimally disturbed (see the remark following Eq. (2)). Because of symmetry between momentum and coordinate, the same is valid also if the coordinate is measured and non-minimally disturbed. The conclusions are however not justified if the oscillator is continuously measured but according to another model.

It is also interesting to evaluate the energy shift caused by the observational procedure. Reading off this shift from Eq.(9) and making use of Eq. (11) we obtain

$$
-i \frac{\kappa \hbar}{4}\left(\hat{l}^{\dagger 2}-\hat{l}^{2}\right)=\frac{\lambda \omega}{4}(\hat{P} \hat{Q}+\hat{Q} \hat{P}) .
$$


Combining this with the Hamiltonian (10) we obtain

$$
\begin{aligned}
\hat{H}_{\mathrm{eff}} & =\frac{1}{2}\left(\hat{P}^{2}+\omega^{2} \hat{Q}^{2}\right)+\frac{\lambda \omega}{4}(\hat{P} \hat{Q}+\hat{Q} \hat{P}) \\
& =\frac{1}{2}\left(\hat{P}+\frac{\lambda \omega}{2} \hat{Q}\right)^{2}+\frac{1}{2}\left(\omega^{2}-\frac{\lambda^{2} \omega^{2}}{4}\right) \hat{Q}^{2} .
\end{aligned}
$$

As $\lambda \omega$ is the friction coefficient, the new oscillational frequency is just the correctly shifted one for a damped oscillator:

$$
\Omega=\sqrt{\left(\omega^{2}-\frac{\lambda^{2} \omega^{2}}{4}\right)} .
$$

Thus we find a consistent description of the ordinary Brownian motion process in terms of continuous measurements.

\section{Conclusion}

Non-minimally disturbing continuous quantum measurements were shown to lead to dissipation of the measured system. The resulting model of dissipation is characterized by a Lindblad master equation and thus avoids the difficulties arising in other approaches. Although only measuring a single observable has been considered in the present paper, generalization on the case of measuring two or more observables is straightforward.

\section{ACKNOWLEDGEMENT}

One of the authors (M.B.M.) acknowledges support from the Royal Swedish Academy of Sciences and the KTH where the main part of this work was completed. The work was supported in part by the Deutsche Forschungsgemeinschaft and Russian Foundation of Basic Research. 


\section{References}

[1] D.Giulini, E.Joos, C.Kiefer, J.Kupsch, I.-O.Stamatescu and H.D.Zeh, Decoherence and the appearance of classical world in quantum theory, Springer, Berlin, 1996.

[2] M.B.Mensky, Quantum measurements and decoherence, Kluwer, Dordrecht, 2000 (Russian translation: Fizmatlit, Moscow, 2001).

[3] A.O.Caldeira and A.J.Leggett, Ann. Phys. 149, 374 (1983); W.H.Zurek, Phys. Rev. D 26, 1862 (1982); W.G.Unruh and W.H.Zurek, Phys. Rev. D 40, 1071 (1989).

[4] V.Ambegaokar, Ber. Bunsenges Phys. Chem., 95, 400 (1991).

[5] W.J.Munro and C.W.Gardiner, Phys. Rev. A 53, 2633 (1996); S.Gnutzmann and F.Haake, Z. Phys. B 101, 263 (1996).

[6] G.Lindblad, Commun. Math. Phys., 48, 119, 1976.

[7] S.Stenholm, in Quantum dynamics of simple systems, Editors G.L.Oppo, S.M.Barnett, E.Riis and M.Wilkinson, Scottish Universities Summers School in Physics and Institute of Physics Publishing, Bristol, 1996, p.304.

[8] M.R.Gallis, Phys. Rev. A 48, 1028 (1993).

[9] L.Diósi, Europhysics Letters, 30, 63 (1995).

[10] A.Konetchnyi, M.Mensky and V.Namiot, Phys. Lett. A 177, 283 (1993). 